\title{
Correção de Atenuação para Sistemas SPECT e PET
}

As imagens obtidas na cintilografia são formadas por fótons que atravessam os diferentes tecidos do corpo humano e alcançam o detector. A probabilidade de este fenômeno ocorrer se reduz com o aumento da probabilidade de interação destes fótons com o tecido. À medida que a nossa população está ficando mais obesa, a apreciação deste fenômeno tem uma grande significância clínica. As principais interações que contribuem para a degradação da imagem em cintilografia e conseqüente atenuação são os efeitos fotoelétricos e 0 espalhamento Compton $^{1}$ (figura 3 ).

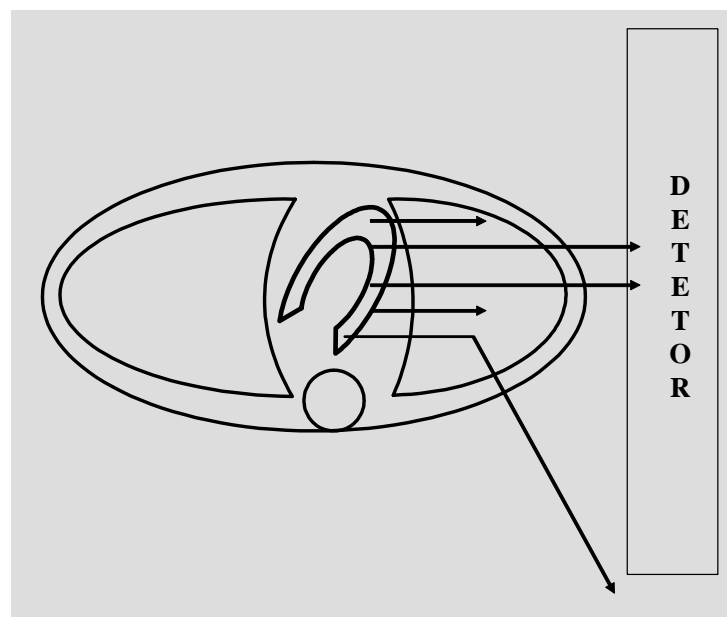

Figura 3 - Modelo esquemático mostrando a emissão de radiação pelo coração. Parte dos fótons emitidos não deixa o corpo do paciente por interagir totalmente com os tecidos (efeito fotoelétrico) e parte interage parcialmente com desvio de sua trajetória e perda de parte da sua energia (efeito Compton). Tanto o efeito fotoelétrico como o Compton são responsáveis pela atenuação dos fótons emitidos. $O$ efeito de atenuação tende a ser mais expressivo em tecidos mais densos, como as estruturas ósseas.

A fração de fótons atenuada é dada pelo coeficiente de atenuação linear $(\mu)$, definido pelo número de fótons que sofrem interação por unidade de comprimento (espessura do atenuador). O coeficiente de atenuação $(\mu)$ é dependente da energia da radiação, da densidade do tecido (número de elétrons por grama) e, para o efeito fotoelétrico, do número atômico. Exemplificando: para fótons com energia de $511 \mathrm{keV}$ os valores de $\mu$ para tecidos moles (densidade próxima à da água), osso e pulmão são respectivamente: 0,095/cm, 0,12/cm e $0,03 / \mathrm{cm}$; para fótons com energia de $140 \mathrm{keV} 0$ coeficiente de atenuação na água é de $0,15 / \mathrm{cm}$, superior àquela dos fótons de $511 \mathrm{keV}(0,095 / \mathrm{cm})$. Matematicamente, a fração de fótons que será transmitida (FT) através de um atenuador segue a equação $\mathrm{FT}=\mathrm{e}^{-\mu \mathrm{x}}$, onde $\mu$ é a soma dos coeficientes de atenuação de cada tecido e $x$ é a espessura do atenuador. $^{2}$ No tórax a atenuação não se faz de modo uniforme devido aos diferentes tipos de tecidos e à grande variabilidade individual. Desta forma, torna-se necessário o conhecimento dos coeficientes de atenuação e das diferentes densidades de tecidos de cada paciente, podendo-se criar um mapa de atenuação a partir de uma fonte externa que irradia o paciente e cria projeções de transmissão da anatomia individual ${ }^{3}$. Algoritmos utilizam estas informações na reconstrução das projeções de emissão, obtendo-se imagens corrigidas para a atenuação. Com a rápida evolução dos computadores, vários sistemas de correção de atenuação foram desenvolvidos, motivados pelo grande impacto dos artefatos de atenuação na prática clínica e diminuição da especificidade do método. No entanto, estes sistemas foram implementados na prática clínica sem validação definitiva, baseados em número insuficiente de estudos clínicos ${ }^{4}$. A implementação destes sistemas requer o manuseio adicional de fontes radioativas e rigoroso controle adicional de qualidade. A pequena experiência até o momento e a relação custo/efetividade desfavorável devem ser motivo de especial atenção em nosso meio, necessitando-se de mais estudos e desenvolvimento tecnológico. Considera-se ainda em estágio primário de apreciação o controle de qualidade para correção de atenuação, com a introdução obrigatória de questões técnicas adicionais, que devem ser satisfatoriamente e sistematicamente observadas, para garantir a qualidade das imagens obtidas ${ }^{5}$. A American Society of Nuclear Cardiology juntamente com a Society of Nuclear Medicine, recentemente, se posicionaram favoráveis ao uso de correção de atenuação juntamente com o gated-SPECT nos exames de perfusão miocárdica para incrementar a acurácia diagnóstica. Definiram prérequisitos para a incorporação do método na prática clínica: 1) suficiente estatística de contagem nos mapas de transmissão; 2) controle de qualidade das imagens adquiridas de modo seqüencial; 3) utilização conjunta de outros fatores de correção; 4) aquisição de imagens capturadas com ECG (gated); 5) treinamento especial dos profissionais para aquisição, processamento e interpretação; 6) interpretação separada das imagens com e sem correção de atenuação ${ }^{6}$.

Contudo, em nosso meio, é preciso cautela para utilizar correção de atenuação, principalmente pela dificuldade de se manterem as fontes radioativas com atividade suficiente para obedecer ao pré-requisito 1) acima, para, de fato, fazer a correção sem introduzir artefatos.

\section{Controle de qualidade}

\section{Equipamento}

Configurações existentes no comércio para criação de imagens tomográficas de transmissão-emissão em 
sistema SPECT: ${ }^{4,5,7}$

- sistemas com fonte linear de varredura, colimador paralelo e dois detectores a $90^{\circ}$;

- arranjo com múltiplas fontes lineares de gadolínio153, colimador paralelo e dois detectores a $90^{\circ+}$;

- sistemas com fonte pontual de varredura (bário133), para 2 ou 3 detectores;

- fonte de transmissão de raios $X$, para 2 detectores ( ${ }^{*}$ controle de qualidade não incluído na diretriz);

Sistemas que utilizavam colimador fan-beam para imagens de transmissão e emissão não estão mais disponíveis no mercado ${ }^{8}$.

Os sistemas com fonte linear de varredura são os mais empregados, já avaliados por estudo clínico multicêntrico ${ }^{9}$. São ou foram utilizados pelas indústrias: GE "AC for Optima", ADAC/Philips "Vantage", "VantagePro/ EXSPECT", SMV "TAC", Elscint "TransACT"4. A varredura de transmissão abrange praticamente todo o campo visual da câmara de cintilação, com a movimentação mecânica da fonte colimada de gadolínio-153 a cada ângulo, para a aquisição das imagens de transmissão. Uma janela eletrônica em sincronia com a linha de varredura percorre o campo visual para aceitar apenas os fótons no fotopico do gadolínio-153 que estejam sendo detectados naquele local ${ }^{10}$. Estes sistemas, com colimador paralelo, requerem o uso de fontes com atividade alta, para adequada estatística de contagem nas imagens de transmissão e estão mais sujeitos à instabilidade mecânica no hardware de varredura ${ }^{5}$. A aquisição conjunta de imagens com gating (sincronização com o ciclo cardíaco pelo intervalo RR do eletrocardiograma) pode ser afetada por rejeição de batimentos, embora este problema não seja comum nos métodos de correção de atenuação utilizados atualmente.

\section{Procedimentos para o CONTROLE DE QUALIDADE}

Energia do fotopico (diário): Através do analisador da altura de pulso, obtém-se a informação se os fótons estão sendo registrados nas janelas apropriadas de energia de emissão, transmissão e espalhamento. Faz-se a abertura manual da fonte de transmissão ou uma varredura vazia (sem o paciente). As orientações do fabricante devem ser consideradas, já que alguns sistemas apresentam diferentes requisitos.

Mecanismos da fonte de transmissão (diário): Para verificar o funcionamento dos mecanismos de abertura e movimentação da fonte, uma aquisição de transmissão vazia (sem o paciente) é realizada, padrão de referência. As imagens planas devem ser inspecionadas para artefatos grosseiros de não uniformidade, como áreas frias, perda de dados, descontinuidades no sentido axial, mudanças abruptas na intensidade dos pixe/s. Sistemas mais estáveis podem ser avaliados semanalmente.
Avaliação da fonte (mensal): É obtida através do RTC que é definido como a razão entre fótons primários de transmissão pela "contaminação" cruzada de fótons scatter de emissão, na janela de energia de transmissão (RTC). Quanto menor a RTC, maior será o viés e ruído na reconstrução dos mapas de atenuação. São causas de baixa RTC: decaimento das fontes de transmissão, atividades elevadas para imagens de emissão, pacientes de grande porte (obesos) ${ }^{10}$. Deve-se obter um padrão de RTC na instalação ou troca de fontes. Estes controles de qualidade podem ser realizados com o fantoma cilíndrico ou antropomórfico torácico, conforme protocolos específicos. Problemas decorrentes: expiração do tempo de validade da fonte, correção inapropriada da "contaminação" cruzada, inconsistência entre transmissão e a varredura vazia.

\section{Controle de qualidade clínica PARA PROCEDIMENTOS EM PACIENTES}

Os parâmetros de RTC são influenciados pelo tamanho do paciente e dose administrada, como acima mencionado. Alguns sistemas permitem a avaliação quantitativa da RCT em projeção plana, previamente à aquisição das imagens de transmissão e emissão, possibilitando correções, como o aumento do tempo ou cancelando a aquisição, conforme valores obtidos de $\mathrm{RCT}^{7,10}$. A avaliação visual, após a reconstrução dos mapas de atenuação, deve ser encorajada ${ }^{3}$ para a identificação de:

- Truncation: artefatos brilhantes em formato de anel na periferia do tórax;

- Contaminação: redução na intensidade da imagem em regiões do coração ou fígado;

- Estudos de baixa contagem estatística: maior incidência de ruído.

\section{Spect com Correção de Atenuação \\ Parâmetros de Aquisição}

Fotopico - As janelas de energia devem ser ajustadas conforme as energias dos fótons de transmissão e emissão. Alguns sistemas ainda recomendam um terceiro fotopico para melhor discriminação do efeito de espalhamento ou scatter. A fonte de transmissão mais utilizada é o gadolínio-153, com duas energias de interesse: $97 \mathrm{keV}$ (28\%) e $103 \mathrm{keV}(20 \%)$, sendo que uma janela de energia de aproximadamente $20 \%$ deve ser centrada em 100 $\mathrm{keV}$, para a captação de ambos os fótons.

Colimador - Atualmente, a maioria dos sistemas utiliza colimador paralelo $0^{4,5,7}$, sendo que a escolha entre colimador de baixa energia e alta resolução ou all purpose, deve basearse no radionuclídeo utilizado para as imagens de emissão.

Órbita de aquisição e projeções - Pode-se utilizar órbita de $180^{\circ}$, circular ou não, em praticamente todos os 
sistemas existentes no comércio. O número de projeções independe da realização de correção de atenuação.

Modo de aquisição - A aquisição tomográfica pode ser realizada de três maneiras ${ }^{7}$ :

a) aquisição seqüencial: duas aquisições independentes para transmissão e emissão (com a fonte de transmissão fechada).

- vantagens: não "contaminação" das imagens de emissão com fótons originários da fonte de transmissão, aquisição com gating. No período em que a fonte de transmissão está fechada, é possível estimar-se a "contaminação" cruzada que ocorre na janela energética de transmissão dos fótons originários da emissão.

- desvantagens: duas aquisições com aumento no tempo total do exame, artefatos de desalinhamento por movimentação do paciente/coração entre as aquisições.

b) aquisição simultânea: ambas as imagens de transmissão e emissão são adquiridas durante o mesmo tempo em cada projeção e na mesma rotação.

- vantagens: tempo menor (única aquisição), com o registro dos fótons alinhados no tempo e espaço ${ }^{10}$, possibilidade de aquisição com gating, se utilizadas duas fontes de gadolínio-153 para a varredura ${ }^{11}$;

- desvantagens: possível contaminação das imagens de emissão com fótons provenientes da fonte de transmissão.

c) aquisição simultânea intercalada: as imagens de transmissão e emissão são adquiridas seqüencialmente em cada projeção, porém na mesma rotação. Metodologia utilizada nos sistemas com capacidade de varredura.

- vantagens: aquisição única, o registro de fótons na mesma projeção diminui erros de desalinhamento, não "contaminação" das imagens de emissão, aquisição com gating. As desvantagens dos outros modos de aquisição são evitadas com este método.

Matriz e tamanho de pixel - Empregam-se a mesma matriz de estudos convencionais de perfusão, $64 \times 64$, e tamanho de pixel de $6.4 \pm 0.2 \mathrm{~mm}$. Não se recomenda a magnificação das imagens de transmissão pela probabilidade do efeito de truncation.

Tempo de aquisição por projeção - 0 tempo total de aquisição deve ser primariamente baseado na estatística de contagem das imagens de emissão, levando-se em consideração o conforto do paciente e a relação sinal/ ruído das projeções de transmissão. Esta última, como previamente mencionado, sofre influência da "idade" da fonte de transmissão e peso do paciente, pois um menor número de fótons irá alcançar o detector. A baixa estatística de contagem nas imagens de transmissão traduz-se em aumento de ruído nas imagens de emissão ${ }^{7,10}$. O fabricante deve fornecer o padrão de contagem para as projeções de transmissão em cada situação, com especial atenção ao momento de troca das fontes de transmissão.

Posicionamento do paciente - No centro da órbita de aquisição e no centro do campo visual da câmara de cintilação, para a redução de artefatos nos mapas de transmissão devidos ao efeito de truncation ${ }^{3,7}$.

Exames sincronizados com o sinal eletrocardiográfico (gating) - Os sistemas que não irradiam todo o campo visual, como os de varredura linear e os de fonte pontual, podem ser afetados pela rejeição de batimentos ou janelas de variabilidade de R-R menor que $100 \%$.

\section{ReConstruÇão e PROCESSAMENTO DE IMAGEM}

Parâmetros comuns aos diferentes sistemas de correção de atenuação para a formação de imagens:

- correção da "contaminação" cruzada de fótons scatter, provenientes da emissão nas projeções de transmissão;

- reconstrução dos mapas de atenuação a partir das projeções de transmissão;

- reconstrução das imagens de emissão corrigidas com o mapa de atenuação;

- utilização de filtros nas imagens de emissão corrigidas.

Correção da "contaminação" cruzada - Normalmente, realizada de modo automático, independente do operador, sendo que a maneira de correção varia conforme as diferentes geometrias utilizadas para transmissão e emissão.

Reconstrução - Realizada em dois estágios ${ }^{8}$ :

a) reconstrução das projeções de transmissão, por retroprojeção filtrada ou por métodos iterativos. A retroprojeção filtrada tem a vantagem da rapidez, mas não modela as estatísticas de contagem e, em certas situações, pode aumentar o ruído nas imagens. Os métodos iterativos são indicados em estudos com baixa estatística de contagem (pacientes muito obesos, tempo curto de aquisição e fonte enfraquecida) e em sistemas mais suscetíveis ao fenômeno de truncation;

b) correção das projeções de emissão a partir do mapa de correção previamente reconstruído. Existem dois métodos de reconstrução para a correção de atenuação: aproximado (método iterativo de Chang) e o estatístico (algoritmos iterativos). Os métodos estatísticos de reconstrução seguem modelos de distribuição de Poisson e Gauss. Exemplos de algoritmos iterativos: weighted least squares (WLS), maximum likelihood-expectation maximization (ML-EM) e ordered subset-expectation maximization (OS-EM). Os algoritmos iterativos estatísticos têm a capacidade de adicionar informações para correção de scatter e fótons de dispersão (distância versus resolução). Estes diferentes métodos devem ser utilizados conforme orientação do fabricante.

Filtros - Os filtros devem ser aplicados após a reconstrução das imagens, de acordo com a orientação do fabricante para cada sistema. A utilização em fase prévia à reconstrução invalida o método. 


\section{REVISÃo DE IMAGEM}

Na ausência de um sistema completamente validado e eficaz de correção de atenuação, as imagens corrigidas devem ser analisadas em conjunto com as imagens não corrigidas, como um instrumento adicional para aumentar a confiança do médico em avaliar artefatos que ele aprendeu a compensar mentalmente, além de habituarse com os novos padrões de distribuição (maior uniformidade de contagem nas paredes do miocárdio), nas imagens corrigidas ${ }^{4,5,8}$.

\section{REFERÊNCIAS}

1. King MA, Tsui BMW, Pan T-S. Attenuation compensation for cardiac single-photon emission tomography imaging: part 1 . Impact of attenuation and methods of estimating attenuation maps. J Nucl Cardiol 1995; 2: 513-24.

2. King MA, Tsui BMW, Pan T-S. Correction of attenuation and scatter for single-photon emission computed tomography. In: Zaret and Beller, Nuclear Cardiology: State of the Art and Future Directions, $2^{\mathrm{a}} \mathrm{ed}$. St Louis: Mosby, 1999; 125-136.

3. Miles J, Cullom SJ, Case JA. An introduction to attenuation correction. J Nucl Cardiol 1999; 6:449-57.

4. Corbett JR, Ficaro EP. Clinical review of attenuation-corrected cardiac SPECT. J Nucl Cardiol 1999; 6: 54-68.

5. Hendel RC, Corbett JR, Cullom SJ et al. The value and practice of attenuation correction for myocardial perfusion SPECT imaging: a joint position statement from the American Society of Nuclear Cardiology and the Society of Nuclear Medicine. J Nucl Cardiol 2002 9: $135-43$.

6. Heller GV, Links J, Bateman TM et al. American Sciety of Nuclear Cardiology and Society of Nuclear Medicine joint position statement: attenuation correction of myocardial perfusion SPECT scintigraphy. J Nucl Cardiol 2004; 11:229-30.

7. Imaging Guidelines for Nuclear Cardiology Procedures- part 1. J Nuc Cardiol, 2001; 8: G1-58.

8. Bar Harbor 2000 panel summaries. J Nucl Cardiol, 2001; 8: 224-316.

\section{Quantificação}

O objetivo da correção de atenuação é diminuir a grande variabilidade na distribuição de contagens nas paredes do miocárdio e não criar um mapa polar absolutamente unifor$\mathrm{me}^{12-14}$. As diferenças na espessura das paredes miocárdicas (efeito de volume parcial) e efeitos de movimentos continuarão a influenciar na intensidade de contagem das imagens. Portanto, a utilização de banco de dados "normal" com correção de atenuação será necessária, possivelmente sexo-independente (igual para homens e mulheres), devido à maior uniformidade na distribuição do traçador $r^{4,8,15}$.

9. Hendel RC, Bermann DS, Cullom SJ et al. Multicenter clinical trial to evaluate the efficacy of correction for photon attenuation and scatter in SPECT myocardial perfusion imaging. Circulation 1999; 99: 2742-49.

10. Galt JR, Cullom SJ, Garcia E.V. Attenuation and scatter compensation in myocardial perfusion SPECT. Semin Nucl Med 1999; 29: 204-20.

11. Bateman TM, Cullon SJ. Attenuation correction single-photon emission computed tomography myocardial perfusion imaging. Semin Nucl Med 2005; 35:37-51.

12. Ficaro EP, Fessler JA, Shreve PD et al. Simultaneous transmission/ emission myocardial perfusion tomography. Diagnostic accuracy of attenuation-corrected 99mTc-sestamibi single-photon emission computed tomography. Circulation 1996; 93: 463-73.

13. He ZX, Scarlett MD, Mahmarian JJ et al. Enhanced accuracy of defect detection by myocardial single-photon emission computed tomography with attenuation correction with gadolinium-153 line sources: evaluation with a cardiac phantom. J Nucl Cardiol 1997; 4: 202-10.

14. Prvulovich EM, Lonn AH, Bomanji JB et al. Effect of attenuation correction on myocardial thallium-201 distribution in patients with low likelihood of coronary artery disease. Eur J Nucl Med 1997; 24: 266-75.

15. Grossman G, Garcia EV, Bateman TM et al. Quantitative technetium99m-Sestamibi attenuation corrected SPECT: development and multicenter trial validation of myocardial perfusion stress genderindependent normal database in an obese population. J Nucl Cardiol 2004; 11:263-72. 